\title{
Randomized study of the effects of quantum resonance technology application on human psychophysiological parameters
}

\begin{abstract}
Objectives: The aim of this study was to demonstrate the beneficial effect of the Quantum Resonance Technology Yippi Wave 3.0 mobile application on the human psychophysiology.

Design: An open, randomized, placebo - controlled trials for two weeks. Participants were measured initially, randomly divided into two groups (test and control) and measured two weeks later after listening to programs twice a day: experimental group - Yippi Wave 3.0 audio mobile application; control group - classical music.

Subjects: 50 apparently healthy adults, age $22-56$ aged, 18 men and 32 women, randomly divided into two groups of 25 people. Randomization was based on the principle of random numbers. The study protocol was approved by the ethics committee of the North-Western Medical University, St. Petersburg, Russia. All participants signed an informed consent form, where a written and oral explanation of the research protocol was provided.
\end{abstract}

Outcome measures: Physical examination by a therapist, psychological testing, rhythmocardiography, biofield parameters analysis.

Results: Participants using Quantum Resonance Technology (Yippi Wave 3.0) apps for two weeks demonstrated positive changes of psychophysiology parameters, while the participants in the control group demonstrated a negative trend of these parameters.

Conclusions: Quantum Resonance Technology (Yippi Wave 3.0) applications have wellness and energized effects on humans.

Keywords: psychophysiology, quantum resonance, bio-well, HRV, open, randomized, placebo - controlled
Volume 7 Issue 3 - 2020

\author{
Korotkov KG,' Churganov OA, ${ }^{2}$ Gavrilova \\ $\mathrm{EA}^{2}$ \\ 'Saint-Petersburg Scientific-Research Institute for Physical \\ Culture, Russia \\ ${ }^{2}$ North-Western Medical University, Russia
}

Correspondence: Korotkov KG, Federal State Budget Institution "Saint-Petersburg Scientific-Research Institute for Physical Culture", Ligovski prospect 56, NIIFC, St. Petersburg, Russia, 199000, Tel +79219368394, Email korotkov2000@gmail.com

Received: April 18, 2020 | Published: May 05, 2020
Abbreviations: HRV, heart rate variability; EPI, electrophotonic imaging; CCD, charge-coupled device

\section{Introduction}

In our previous papers we demonstrated the combined effect of music and frequency therapy ${ }^{1}$ as well as the effect of Quantum Resonance Technology (Yippi Wave 3.0) applications on water, ${ }^{2}$ environmental sensor ${ }^{3}$ and protective effect to the influence of mobile phone to people. ${ }^{4}$ In this paper we present results of the randomized study of the effects of this technology on human psychophysiological parameters.

\section{Methods}

This was an open, randomized, placebo - controlled research for two weeks. People were measured initially, randomly divided into two groups and measured two weeks later after listening to Yippi Wave 3.0 mobile application or classical music twice a day. This study was approved by the Institutional Review Board (IRB) of the NorthWestern Medical University, St. Petersburg, and the protocols used in the study were approved by the Committee of Human Subjects Protection of the IRB of the North-Western Medical University, St. Petersburg. The official trial registration number is $56 / 01 / 2020$ (Protocol N 56 from 01.15.2020 of the meeting of the IRB of the North-Western Medical University, St. Petersburg, Russia).

50 apparently healthy adults, age $24-56$ aged $(43.8+9.5$ years $)$, 18 men and 32 women, were randomly divided into two groups of
25 people. Sample size were calculated in accordance with ${ }^{5}$ with confidence level 0.95 and desired power 0.8. Randomization was based on the principle of the random numbers. All participants signed an informed consent form, where a written and oral explanation of the research protocol was provided. Subjects were screened for wellness; exclusion criteria included conditions that could compromise physical and mental health, such as:

a. severe chronic diseases,

b. acute somatic, neurological diseases,

c. receiving medicines and dietary supplements during the experiment,

d. clinically significant changes in functional indicators that indicate undiagnosed disease and require additional screening,

e. clinically detectable mental pathology.

All participants had a physical examination by a therapist, psychological testing, rhythmocardiography, biofield parameters analysis.method. Participants in the control group for two weeks, twice a day were listening to classical music; participants in the experimental group for two weeks, twice a day were listening to Quantum Resonance Technology apps (Yippi Wave 3.0) audio via their mobile phones. After this, all the tests were repeated with the same protocol as in the first trial.The following techniques have been used in the study: psychological testing; Bio-Well GDV method, and heart rate variability (HRV). Questionnaire "State of health, activity, 
mood" is intended for self-assessment of the following psychological qualities: a feeling of well-being, activity, mood. Psycho-diagnostics of the subjects was carried out as follows. The control and experimental group read 30 pairs of opposite definitions, from which they had to choose a more suitable condition on a scale from 0 to 3 points. Interpretation of indicators according to "FAM" method was carried out by comparing the state with a scale of 322101123 . For example, between the statements "Feeling strong" and "Feeling weak" there are numbers 321011123 . The number " 0 " means a neutral state in which the person cannot be classified whether he/she is either weak or strong. The number " 3 " means that the individual feels very strong and vice versa. In this test, all lines are considered and evaluated separately.

The technique of Electrophotonic Imaging (EPI) allows the recording of electron and photon emission stimulated by an electromagnetic field in any subject, as well as the acquisition of these data by computer image processing. The short electric impulse (10 microsec) on the camera plate stimulates subjects and generates a response in the form of an excited gas plasma (that is why in physical terms this approach is known as Gas Discharge Visualization - GDV ${ }^{7}$ $\left.{ }^{10}\right)$. This plasma emits light which is directly measured by a chargecoupled device (CCD), the state of the art in measuring low-level light that is used in astrophysics and other scientific endeavors. The CCD registers the pattern of photons detected over time. These digital data are transmitted directly into a computer for data processing, and each image from the light emitted is stored as a graphics file. These two-dimensional images of the light are then used to calculate the area, emission intensity, fracticality, and other parameters. The EPI technique has been found to be effective in evaluating the state of individual human health, ${ }^{11-14}$ and in the monitoring of individual reactions to different kinds of training. ${ }^{11-17}$ Measurement of heart rate variability (HRV) by the rhythmogram was performed at rest using the "Neurosoft" device when analyzing 5-minute records of heart rate variability. In interpreting the data, the standards for evaluating HRV developed by the European Heart Society and the North American Society of Electrophysiology ${ }^{18,19}$ has been used. Statistical processing of data was done using SPSS Statistics software.

\section{Results}

\section{Psychological testing}

The data of psychological status according to the method "State of health, activity, mood" is presented in Table 1. As we can see from these data, the control group parameters dropped down except for activity, while in the experimental group we can see a statistically significant increase of all parameters after two weeks of listening to Quantum Resonance Technology apps (Yippi Wave 3.0) audio program, which indicates a better mood state.

Table I Data of the self-evaluation by the method "State of health, activity mood" in the control and experimental groups

\begin{tabular}{lllll}
\hline Indicator & \multicolumn{2}{l}{ Control group } & \multicolumn{2}{l}{ Experimental group } \\
\hline & I test & 2 test & I test & 2 test \\
\hline Feeling & $50.7 \pm 11.0$ & $46.3 \pm 8.8$ & $54.9 \pm 5.5$ & $58.1 \pm 4.9$ \\
Activity & $46.4 \pm 10.1$ & $49.6 \pm 11.8$ & $50.9 \pm 5.6$ & $63.3 \pm 3.4^{* * *}$ \\
Mood & $51.9 \pm 10.8$ & $48.2 \pm 10.0$ & $57.9 \pm 4.3$ & $64.5 \pm 3.4 * * *$ \\
General & $49.7 \pm 9.1$ & $48.0 \pm 9.5$ & $54.6 \pm 4.1$ & $62.0 \pm 3.3 * * *$ \\
\hline ***
\end{tabular}

***-p<0.00I

\section{HRV data}

The rhythmogram was recorded at rest and evaluated by 5 -minute recording in rest. Dynamics of median parameters of HRV in two groups is presented in Table $2 \&$ Figure 1. As it follows from the data, the experimental group was characterized by the growth of parameters reflecting parasympathetic activity and variability of heart rhythm and the decrease of parameters which reflect the degree of sympathetic regulation and centralization of heart rhythm. Significant changes were noted in most of the indexes after two weeks of listening to Quantum Resonance Technology apps (Yippi Wave 3.0) program, which indicated growth of the economy of respiratory and cardiovascular systems functioning, growth of the adaptive and energetic potential of the organism, as well as a recovery potential. Statistically significant changes were detected on the Stress-index, which correlates with the results of psychological testing. In the control group, inverse changes in HRV were noted, indicating increased activity of the sympathoadrenal system, which reflected the centralization of the heart rhythm, reduction in the adaptation potential of the organism and its reserve capacity. Perhaps, this is due to the time of the experiment: the end of the year, which is why summing up, submitting reports and a high level of tension of both the psycho-emotional and the regulatory activity.

Table 2 Dynamics of median variables in the control and experimental groups

\begin{tabular}{|c|c|c|c|c|}
\hline \multirow[t]{2}{*}{ Indicator } & \multicolumn{2}{|c|}{ Control group } & \multicolumn{2}{|c|}{ Experimental group } \\
\hline & I test & 2 test & I test & 2 test \\
\hline RR (msec) & $968.6 \pm 147$ & $929.8 \pm 148.8$ & $932.6 \pm 112.3$ & $904.7 \pm 113.8$ \\
\hline LF/HF & $1.1 \pm 0.9$ & $1.6 \pm 1.6$ & $1.6 \pm 1.1$ & $1.7 \pm 1.5$ \\
\hline Mo (msec) & $953.9 \pm 158.6$ & $930.3 \pm 160.4$ & $911.3 \pm 114.8$ & $872.5 \pm 111.2$ \\
\hline TP $\left(\mathrm{msec}^{2}\right)$ & 2578 & 2062 & $117 \mid$ & $1590 *$ \\
\hline CV\% & $5.8 \pm 2.2$ & $6.2 \pm 3.1$ & $4.6 \pm 1.7$ & $6.1 \pm 2.8 * *$ \\
\hline $\operatorname{VLF}\left(\mathrm{msec}^{2}\right)$ & 673 & 752 & 470 & $589 *$ \\
\hline HF \% & $54.8 \pm 18.8$ & $44.8 \pm 23.2$ & $45.9 \pm 19.3$ & $45.2 \pm 17.6$ \\
\hline $\mathrm{HF}\left(\mathrm{msec}^{2}\right)$ & 1242 & 591 & 339.5 & 417.5 \\
\hline SI (ratio) & $110.5 \pm 149.9$ & $|2| .2 \pm|4| .6$ & $127.9 \pm 86.5$ & $102.2 \pm 67.9$ \\
\hline SDNN(msec) & $57.1 \pm 24.6$ & $59.6 \pm 34.1$ & $42.3 \pm 13.7$ & $55.2 \pm 29.2^{*}$ \\
\hline
\end{tabular}

$*_{\text {-p }}<0.05$

Changes \%\%

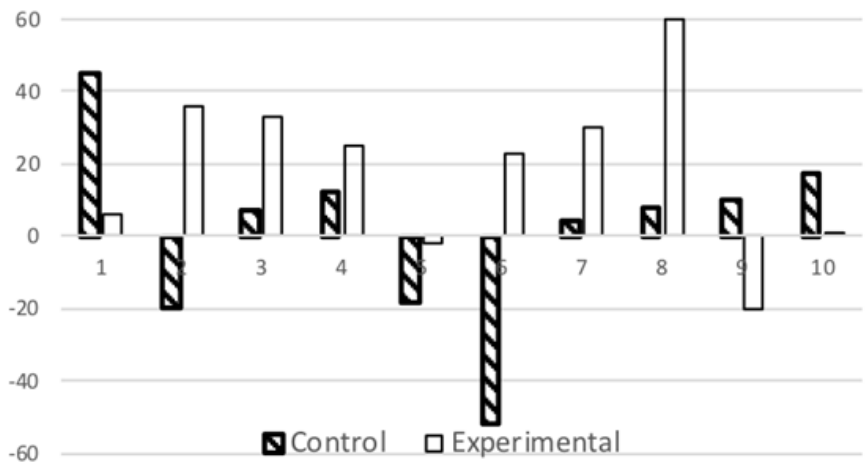

Figure I Relative changes of HRV parameters between $2^{\text {nd }}$ and $\left.\right|^{\text {st }}$ measurements in control and experiment groups.

I, LF/HF; 2,TP; 3, CV\%; 4,VLF; 5, HF \%; 6, HF (msec $\left.{ }^{2}\right) ; 7, \mathrm{SDNN} ; 8, \mathrm{LF}$ norm (\%); 9, SI (ratio); I0, LF. 


\section{Human biofield parameters, bio-well GDV data}

The results are presented in Table 3 \& Figure 2. As we can see from the graphs, in the initial measurement between control and experimental groups there was no difference, but 2 weeks later Energy dropped down in the control group and Stress dropped as well. In the experimental group, Energy increased and Stress decreased more than in the control group. There was no statistical difference between 1 st and 2 nd tests for both groups, but if we removed 2 people from calculations, Energy increase in the experimental group would be statistically significant.For the participants of the control group listening to classic music Stress level dropped down and Energy decreased. Figure Comparison of averaged Bio-Well data for the initial measurement (1) and after 2 weeks (2) for the control and experimental groups for Stress coefficient.

Table 3 Bio-Well GDV indexes the control and experimental groups

\begin{tabular}{lllll}
\hline Parameters & \multicolumn{2}{l}{ Control group } & \multicolumn{2}{l}{ Experimental group } \\
\hline & test I & test 2 & test I & test 2 \\
\hline Stress & $3.24 \pm 0.02$ & $3.23 \pm 0.02$ & $3.24 \pm 0.02$ & $3.12 \pm 0.02 *$ \\
Energy & $57.63 \pm 0.06$ & $55.00 \pm 0.08$ & $57.63 \pm 0.07$ & $57.80 \pm 0.08$ \\
Balance & $96.01 \pm 0.80$ & $95.25 \pm 0.78$ & $96.01 \pm 0.87$ & $96.31 \pm 0.78$ \\
$\begin{array}{l}\text { Fractal } \\
\text { coefficient }\end{array}$ & $2.52 \pm 0.06$ & $2.51 \pm 0.08$ & $2.52 \pm 0.05$ & $2.41 \pm 0.04$ \\
\hline$*_{\mathrm{p}}<0.05$ & & & &
\end{tabular}

Energy, J*10-2

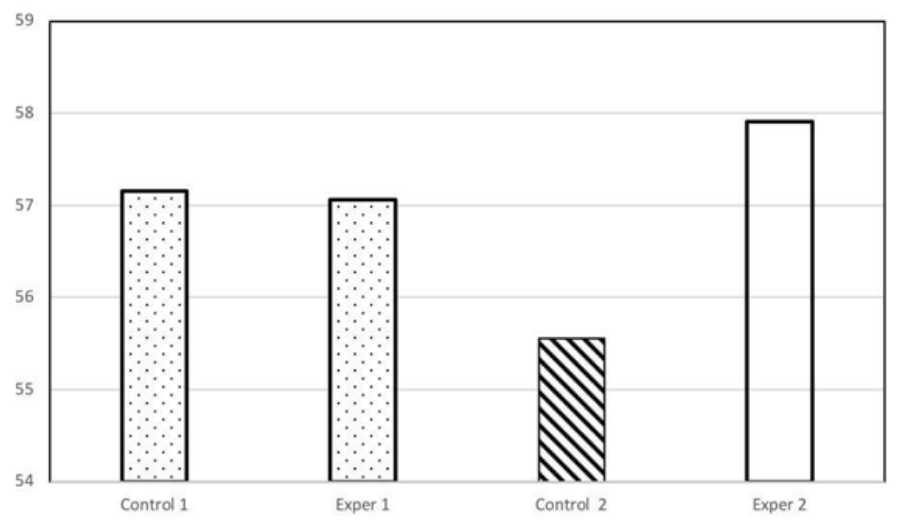

Figure 2 Comparison of averaged Bio-Well data for the initial measurement (I) and after 2 weeks (2) for the control and experimental groups for the Energy parameter.

\section{Discussion}

The aim of this study was to demonstrate the beneficial effect of the Quantum Resonance Technology Yippi Wave 3.0 mobile application on the human psychophysiology. Study was organized as an open, randomized, placebo - controlled trials for two weeks. Participants were measured initially, randomly divided into two groups (test and control) and measured two weeks later after listening to programs twice a day: experimental group - Yippi Wave 3.0 audio mobile application; control group - classical music. According to the software developers (https://yippiweb.com/t-rt-history) their apps are developed based on the principles of subtle energy. Subtle energy is a term that refers to any type of energy that has some empirical scientific support for its existence but nonetheless lies outside of the four forces accepted by mainstream science: the strong and weak nuclear forces, electromagnetism, and gravity. ${ }^{19-21}$ As stated William Tiller, Professor Emeritus of the Department of Materials Science and Engineering at Stanford: "Although subtle energies are frequently assumed to be similar to or associated with electromagnetic fields, this is erroneous. In addition, subtle energies involved in biologic processes are not necessarily weak but rather elusive, because they cannot be measured directly, but only by their effects". He has demonstrated that a subtle energy can be used to increase or decrease the $\mathrm{pH}$ of an aqueous solution by one full unit and to increase the in vitrothermodynamic activity of alkaline phosphatase, a liver enzyme. ${ }^{19}$ Subtle energies involved in acupuncture, homeopathy, and influence of different devices to human psychophysiology reside in what Tiller refers to as the vacuum level of nature. In this paper we tested one more novel approach of using subtle energies with modern technology. When the audio file was turned on, the electromagnetic field was modulated, which affected the parameters of the environment and this has an influence on water ${ }^{1}$ and gas-discharge sensor. ${ }^{2}$ Heart rate variability reflects the effect of control signals that reconfigure cells, organs or systems in the interest of maintaining homeostasis or adapting the body to new conditions of the external and internal environment. The experimental group was characterized by reliable positive dynamics of both statistical and spectral indicators of variability and a decrease in the centralization of the heart rhythm, reflecting the growth of parasympathetic regulation, reserve capacity of the body, as well as the general level of health of the tested.This investigation should be considered as a pilot study, as the study includes different age groups, sexes, and professions included in a small cohort. We hope, that the presented results stimulate the development of new studies, dedicated to the influence of subtle energy on human health and wellbeing.

\section{Conclusion}

Participants using Quantum Resonance Technology (Yippi Wave 3.0) apps for two weeks demonstrated positive changes of psychophysiology parameters, while the participants in the control group demonstrated a negative trend of these parameters. We may conclude that, Quantum Resonance Technology apps (Yippi Wave 3.0) applications used for a prolonged time had energized protective effects for the Human Energy Field, the growth of adaptive abilities and reserve capabilities of the body, as well as general health, even in conditions of psycho-emotional overstrain.

\section{Acknowledgments}

All the authors of this paper contributed in preparing protocol of the study, organizing research process, collecting and processing data, and preparation of this paper in equal parts.

\section{Conflicts of interest}

Authors disclose no commercial associations that might create a conflict of interest in connection with submitted manuscripts.

\section{Funding}

Authors had no funding for this research.

\section{References}

1. Korotkov K. The combined effect of music and frequency therapy from the BioCor device on stress level in humans. International Journal of Complementary \& Alternative Medicine. 2020;13(2):69-72. 
2. Korotkov K. Effect of music on structuration of water. International Journal Alternative Complementary Medicine. 2020;13(1):14-16.

3. Korotkov K. Remote detection of music influence with physical sensor Journal Applied Biotechnology and Bioengineering. 2020;7(1):7-10.

4. Korotkov KG. Influence of mobile phone to people and protective effect of quantum resonance technology. Journal Applied Biotechnology and Bioengineering. 2020;7(2):48-51.

5. Charan J, Biswas T. How to Calculate Sample Size for Different Study Designs in Medical Research? Indian Journal Psychol Med. 2013;35:121126.

6. Undrwood B, Forming W. The Mood Survey: A Personality Measure of Happy and Sad Moods. Journal of Personality Assessment. 1980;44(4):404-407.

7. http://www.sputnik.bio-well.com/

8. Korotkov KG, Orlov DV, Williams BO. Application of Electrophoton Capture (EPI) Analysis Based on Gas Discharge Visualization (GDV) Technique in Medicine: A Systematic Review. Journal Alternative and Complementary Medicine. 2010;16(1):13-25.

9. Korotkov KG. The Energy of Health. Amazon.com publishing. 2017.

10. Korotkov KG. Review of EPI papers on medicine and psychophysiology published in 2008-2018. International Journal of Complementary and Alternative Medicine. 2018;11(5):311-315.

11. Muehsam D, Chevalier G, Barsotti T, An Overview of Biofield Devices. Global Advances in Health Medicine. 2015;4:42-51.

12. Korobka IE, Yakovleva TG, Korotkov KG, et al. Electrophotonic Imaging technology in the diagnosis of autonomic nervous system in patients with arterial hypertension. Journal of Applied Biotechnology and Bioengineering. 2018;5:112-118.
13. Buck KH, Novelli C, Costa FT, et al. The use of bioelectrography in the comparison between women with breast cancer, healthy sedentary women and women who run. Center for Advanced Research on Quality of Life. 2016;8(2):9-11.

14. Yakovleva TG, Buntseva OA, Belonosov SS, et al. Identifying Patients with Colon Neoplasias with Gas Discharge Visualization Technique. Journal Alternative and Complementary Medicine. 2015;21:720-724.

15. Kushwah KK, Nagendra HR, Srinivasan TM. Effect of Integrated Yoga Program on Energy Outcomes as a Measure of Preventive Health Care in Healthy People. Central European Journal of Sport Sciences and Medicine. 2015;12(4):61-71.

16. Shiva KK, Nagendra HR, Srinivasan TM, et al. Electrophotonic Imaging Based Analysis of Diabetes. International Journal Alternative and Complementary Medicine. 2016;4(5):134-137.

17. Sushrutha S, Hegde M, Nagendra HR, et al. Comparative study of Influence of Yajña and Yogāsana on stress level as Measured by Electron Photonic Imaging (EPI) Technique. International Journal Science and Research. 2014;8:1402-1406.

18. Heart rate variability. Standards of measurement, physiological interpretation, and clinical use. Task Force of the European Society of Cardiology and the North American Society of Pacing and Electrophysiology. Heart rate variability. Standards of Measurement. Physiological interpretation and clinical use, Circulation. European Heart Journal. 1996;17:354-381.

19. Tiller W. What is Subtle Energy? Journal Scientific Exploration. $1993 ; 7: 3: 293-304$.

20. Bioelectromagnetic and Subtle Energy Medicine. In: Paul J Rosch, editor. CRC Press, NY; 2015.

21. Morley K. Symbols and Subtle Energy.

22. Korotkov KG. Device for measuring electromagnetic field intensity. 2015 